\title{
NIVEIS SANGUINEOS DE VITAMINA A E CAROTENO EM INDIVIDUOS DE MEIA-IDADE E IDOSOS, EM ONZE LOCALIDADES DO ESTADO DE SÃO PAULO, BRASIL
}

Maria José Roncada*

Maria de Fátima Nunes Marucci*

Isildinha Marques dos Reis Lamonica*

RONCADA, M.J. et al. Niveis sangüíneos de vitamina A e caroteno em indivíduos de meia-ldade e idosos, em onze localidades do Estado de São Paulo, Brasil. Rev. Saúde públ., S. Paulo. $19: 336-43,1985$.

RESUMO: Visando preencher lacuna no conhecimento da hipovitaminose A, no que concerne a estudos bioquímicos em idosos, foram analisados os resultados de dosagens sanguíneas de vitamina A e caroteno em 158 indivíduos de ambos os sexos, de 50 anos e mais, residentes em onze localidades do Estado de São Paulo (Brasil), divididos em 4 grupos etários (50-54, 55-59, 60-64 e 65 e mais anos). Não foram encontradas diferenças significativas para vitamina $A$ entre as médias dos 4 grupos etários, nem entre os dois sexos. Entretanto, com relação ao caroteno sanguíneo, as mulheres apresentaram valores médios mais elevados nos 4 grupos etários, sendo significativa essa diferença. Segundo a classificação do Interdepartmental Committee on Nutrition for National Defense (ICNND), a população estudada não apresentou problema de Saúde Pública com relação à hipovitaminose $\mathrm{A}$.

UNITERMOS: Vitamina A, deficiência. Vitamina A, dosagem sangüínea. Caroteno, dosagem sangüínea. Idosos.

\section{INTRODUÇĀO}

O aumento da população idosa, tanto em números absolutos quanto relativos ${ }^{12}$, trazendo consigo problemas específicos, vem despertando o interesse de estudiosos, inclusive da Organização Mundial da Saúde, que estabeleceu como lema do Dia Mundial da Saúde, em 1982, "Remoçar a Velhice" 15. Este aspecto é extremamente importante, principalmente considerando-se que a longevidade da população em geral tem crescido, acarretando um aumento nos grupos etários mais idosos, e que, no final do século, ela necessitará de programas especiais de nutrição. No caso do Brasil, a vida média aumentou de cerca de 42 anos, em 1940, para cerca de 58 anos, em 1977; no Estado de São Paulo, esse valor é de 61 anos para os homens e 68 para as mulheres ${ }^{14}$.

Enquanto alguns países vêm pesquisando aspectos relacionados a essa área há mais de $25 \operatorname{anos}^{10}$ e até a metodologia de levantamento sobre o consumo alimentar foi alvo de investigação ${ }^{17}$, somente nos últimos anos é que tem havido, entre nós, preocupação pelo assunto $2,3,8,8,9,22$. No nosso meio, o atendimento ao idoso foi iniciado por grupos particulares e entidades religiosas, em conjunto com outras, como o Serviço Social do Comércio (SESC) e o Serviço Social da Indústria (SESI), tendo sido instituído o "Ano Nacional do Idoso", em 1982, pelo Decreto n. ${ }^{\circ} 86.880^{4}$, bem como criada uma Comissão Nacional

\footnotetext{
- Do Departamento de Nutrição da Faculdade de Saúde Pública da Universidade de São Paulo - Av. Dr. Arnaldo, 715 - 01255 - São Paulo, SP - Brasil.
} 
RONCADA, M.J. et al. Níveis sanguíneos de vitamina $\mathbf{A}$ e caroteno em indivíduos de meiaidade e idosos, em onze localidades do Estado de São Paulo, Brasil. Rev. Saúde públ., S. Paulo, 19:336-43, 1985.

para coordenar e apresentar sugestões quanto à problemática dessa faixa da população.

Runcie ${ }^{21}$, assim como McClean e col. ${ }^{16}$ referem a escassez de pesquisa e informações sobre o estado nutricional dos idosos, respectivamente, na GrãBretanha e na Nova Zelândia, situação semelhante à do Brasil.

As necessidades nutricionais dessa faixa etária, quanto a proteínas, gorduras e carboidratos, são bem conhecidas, podendo ser atendidas por dietas habituais. Entretanto, as necessidades fisiológicas relacionadas a vitaminas são mais problemáticas, tendo Runcie ${ }^{21}$ proposto a realização de inquéritos nutricionais globais entre os idosos para que, a partir dos resultados, sejam desenvolvidos para eles programas preventivos formais para esta década.

Segundo Vir e Love ${ }^{27}$, os idosos são um segmento da população bastante vulnerável a problemas nutricionais e que talvez apresente dificuldade em converter a pró-vitamina A para a sua forma ativa.

Devido ao decréscimo da ingestão calórica e alteração no metabolismo dos idosos, alguns autores têm sugerido a vulnerabilidade desse grupo, especialmente com relação a deficiências vitamínicas ${ }^{7,11}$. Baker e col. ${ }^{1}$ reforçam que o processo de envelhecimento pode interferir no metabolismo dos nutrientes $e$ que as pessoas de mais de 60 anos estão propensas a desordens que podem conduzir a carências de vitaminas.

Já em 1948, Kirk e Chieffi ${ }^{13}$ chamavam a atenção para a escassez de dados sobre a concentração plasmática de vitamina $\mathrm{A}$ e carotenos em indivíduos idosos e de meia-idade, apesar da existência de uma extensa literatura sobre os aspectos clínicos da hipovitaminose $\mathrm{A}$.

Como também na literatura científica nacional esse aspecto específico da nutrição não foi abordado, planejamos o presente trabalho com o objetivo de estudar os níveis sanguíneos de vitamina $\mathbf{A}$ e caroteno em adultos de 50 anos e mais, de ambos os sexos e moradores de várias localidades do Estado de São Paulo.

\section{METODOLOGIA}

No período de 1969 a 1973, o Departamento de Nutrição da Faculdade de Saúde Pública da Úniversidade de São Paulo realizou inquéritos nutricionais familiares (clínico, bioquímico e alimentar) em onze localidades paulistas, onde foi feito cadastramento que possibilitou uma amostra representativa da população no que tange a sexo e idade ${ }^{19}$.

Embora a maioria dos demógrafos julgue 65 anos como a idade limite a partir da qual os indivíduos seriam considerados velhos ou idosos, não há consenso entre os autores sobre esse marco inicial, referindo alguns 50 , outros 60 e outros, ainda, 65 anos de idade ${ }^{14}$. Todhunter ${ }^{25}$ considera idoso o indivíduo de 60 anos e mais, embora refira que, segundo Anderson, nenhum indivíduo deveria ser considerado como tal até 80 anos de idade.

Por isso, julgamos conveniente considerar para este estudo os indivíduos a partir dos 50 anos de idade, possibilitando, assim, verificar o comportamento da vitamina $A$ e caroteno sangüíneos também em adultos na meia idade. Portanto, neste trabalho, estudamos o grupo etário de 50 anos e mais daquela amostra ${ }^{19}$, constituído de 158 indivíduos não institucionalizados ( 75 do sexo feminino e 83 do masculino), com idades compreendidas entre 50 e 94 anos, supostamente saudáveis, ou seja, não apresentando patologias evidenciáveis ou que os deixassem acamados ou hospitalizados. Esses indivíduos foram distribuídos em 4 faixas etárias $(50$ a 54,55 a 59,60 a 64 e 65 e mais anos) para verificar se existiriam variações significativas nos níveis dos nutrientes em questão com o avançar da idade. 
RONCADA, M.J. et al. Níveis sanguíneos de vitamina A e caroteno em indivíduos de meiaidade e idosos, em onze localidades do Estado de São Paulo, Brasil. Rev. Saúde públ., S. Paulo, 19:336-43, 1985.

Os resultados das dosagens de vitamina A e caroteno plasmáticos (realizados pelo método de Carr-Price ${ }^{26}$ ) foram expressos por meio de média $(\overline{\mathrm{x}})$ e desvio padrão (DP), e na comparação entre as médias, segundo sexo e idade, foi aplicada a análise de variância a 2 fatores $\operatorname{fixos}^{28}$.

Os critérios para se caracterizar a hipovitaminose A como problema de Saúde Pública e para classificar os resultados das dosagens bioquímicas são os sugeridos pelo "Interdepartmental Committee on Nutrition for National Defense" (ICNND) ${ }^{26}$; por essa classificação os resultados de vitamina $\mathrm{A}$ e caroteno sangüíneos podem ser, respectivamente: $\mathrm{D}$ deficiente $(\leqslant 10 \mu \mathrm{g} / 100 \mathrm{ml} \mathrm{e} \leqslant 20 \mu \mathrm{g} /$ $100 \mathrm{ml}), \mathrm{B}$ - baixo $(10$ a $19,9 \mu \mathrm{g} / 100 \mathrm{ml}$ e 20 a $39,9 \mu \mathrm{g} / 100 \mathrm{ml}), \mathrm{A}$ - aceitável ( 20 a $49,9 \mu \mathrm{g} / 100 \mathrm{ml}$ e 40 a $99,9 \mu \mathrm{g} / 100 \mathrm{ml}$ ) e $\mathrm{H}$ - alto $(\geqslant 50 \mu \mathrm{g} / 100 \mathrm{ml} \mathrm{e} \geqslant 100 \mu \mathrm{g} /$ $100 \mathrm{ml}$ ). O teste de qui-quadrado foi empregado para verificar a existência de homogeneidade na distribuição dos resultados segundo essas classes, individualmente por sexo e idade.

\section{RESULTADOS E DISCUSSÃO}

Os resultados das dosagens de vitamina $\mathrm{A}$ e de caroteno plasmáticos foram analisados segundo sexo e idade.

Inicialmente, calculamos as médias dos resultados de vitamina $\mathrm{A}$ plasmática (Tabela 1 , verificando que nos grupos de 50 a 54 anos e de 55 a 59 anos os valores para o sexo masculino foram mais altos do que para os do sexo feminino, o inverso acontecendo com os indivíduos de 60 anos e mais (Fig. 1). Entretanto, a diferença encontrada não foi significante $(p>0,05)$. Observamos, ainda, entre os indivíduos do sexo masculino um irregular declínio com o aumento de idade, em relação à vitamina $\mathrm{A}$ plasmática.

Thiele e col. ${ }^{24}$, trabalhando com 104 indivíduos de 15 a 65 anos, e dividindo o grupo em 3 categorias etárias (15 a 23, 24 a 34 e 35 a 65 anos) encontraram valores mais baixos entre os homens nas duas primeiras categorias e mais altos para o grupo de 35 a 65 anos; entretanto, não houve diferenças estatisticamente sig-

\section{TABELA 1}

Médias $(\bar{x})$ e desvios-padrões (DP) dos níveis plasmáticos de vitamina $A(\mu \mathrm{g} / 100 \mathrm{ml})$ em 156 indivíduos de 50 anos e mais, segundo sexo e idade, em onze localidades do Estado de São Paulo, 1969-1973.

\begin{tabular}{|c|c|c|c|c|c|c|}
\hline \multirow{3}{*}{$\begin{array}{l}\text { Idade } \\
\text { (anos) }\end{array}$} & \multicolumn{6}{|c|}{ Sexos } \\
\hline & \multicolumn{3}{|c|}{ Masculino } & \multicolumn{3}{|c|}{ Feminino } \\
\hline & $\mathrm{N}$ & $\overline{\mathrm{x}}$ & (DP) & $\mathrm{N}$ & $\overline{\mathrm{x}}$ & (DP) \\
\hline $50-54$ & 26 & 48,80 & $(26,15)$ & 30 & 44,47 & $(24,56)$ \\
\hline $55-59$ & 20 & 46,10 & $(17,27)$ & 12 & 35,12 & $(21,22)$ \\
\hline $60-64$ & 14 & 36,49 & $(17,51)$ & 10 & 49,90 & $(19,63)$ \\
\hline$\geqslant 65$ & 21 & 38,40 & $(14,30)$ & 23 & 39,69 & $(21,62)$ \\
\hline \multicolumn{7}{|l|}{ A nova* } \\
\hline \multicolumn{4}{|c|}{ Efeito devido à idade } & \multicolumn{3}{|c|}{$F=1,05$ n.s. } \\
\hline \multicolumn{4}{|c|}{ Efeito devido a sexo } & \multicolumn{3}{|c|}{$F=0,15$ n.s. } \\
\hline \multicolumn{4}{|c|}{ Efeito devido a idade e sexo } & \multicolumn{3}{|c|}{$F=1,60$ n.s. } \\
\hline
\end{tabular}

* Análise de variância a dois fatores fixos.

n.s.: não significañte $(\mathrm{p}>0,05)$. 
RONCADA, M.J. et al. Níveis sangüíneos de vitamina A e caroteno em indivíduos de meiaidade e idosos, em onze localidades do Estado de São Paulo, Brasil. Rev. Saúde públ., S. Paulo, 19:336-43, 1985.

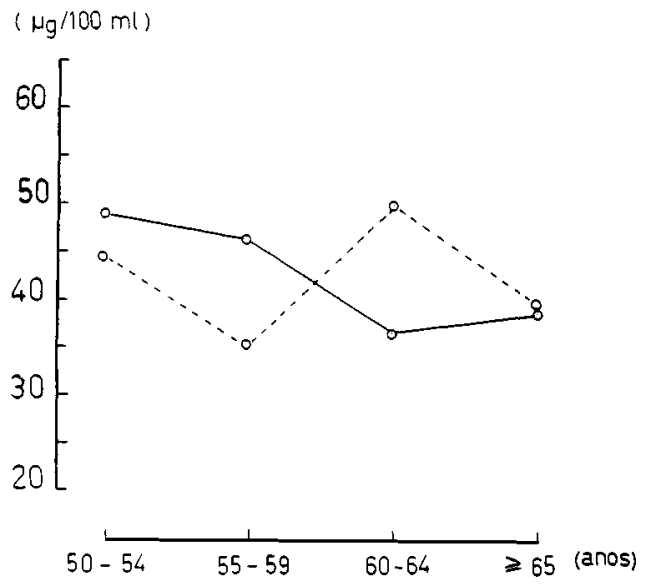

--D-- - FEM.

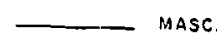

Fig. 1 - Médias das concentraçôes sanguíneas de vitamina $A(\mu \mathbf{g} / 100 \mathrm{ml})$, segundo sexo e idade, em onze localidades do Estado de São Paulo. 1969-1973.

nificantes no grupo, quer quanto a sexo ou idade.

Já Kirk e Chieffi ${ }^{13}$, estudando o comportamento das vitaminas em 155 indi- víduos de meia-idade e idosos (entre 40 e 99 anos), verificaram que não houve diferenças estatisticamente significantes entre os dois sexos, nem entre as médias dos vários grupos etários, embora os valores individuais observados variassem grandemente. O comportamento das médias nos grupos etários de 60 anos e mais, foi semelhante ao obtido no presente trabalho.

Quanto às médias dos resultados de caroteno plasmático (Tabela 2), os valores mostraram-se abaixo daquelas médias consideradas normais por Ross e Parker ${ }^{20}$, para todos os grupos etários e ambos os sexos. Observamos, ainda, que us valores médios para os indivíduos do sexo feminino foram mais elevados do que os do sexo masculino (Fig. 2), sendo esta diferença significativa $(\mathrm{p}<0,01)$. Este fato também tem sido assinalado por outros autores, como Campbell e Tonks ${ }^{5}$, que constataram valores médios para carotenos totais mais altos nas mulheres do que entre os homens, quando estudaram 133 indivíduos normais de ambos os sexos, com idades entre os $18 \mathrm{e}$ 73 anos.

TABELA 2

Médias ( $\overline{\mathrm{x}}$ ) e desvios-padrões (DP) dos níveis plasmáticos de caroteno $(\mu \mathrm{g} / 100 \mathrm{ml})$ em 158 indivíduos de 50 anos e mais, segundo sexo e idade, em onze localidades do Estado de São Paulo, 1969-1973.

\begin{tabular}{|c|c|c|c|c|c|c|}
\hline \multirow{3}{*}{$\begin{array}{l}\text { Idade } \\
\text { (anos) }\end{array}$} & \multicolumn{6}{|c|}{ Sexos } \\
\hline & \multicolumn{3}{|c|}{ Masculino } & \multicolumn{3}{|c|}{ Feminino } \\
\hline & $\mathbf{N}$ & $\overline{\mathbf{x}}$ & (DP) & $\mathbf{N}$ & $\overline{\mathbf{x}}$ & (DP) \\
\hline $\begin{array}{l}50-54 \\
55-59 \\
60-64 \\
\geqslant 65\end{array}$ & $\begin{array}{l}26 \\
20 \\
15 \\
22\end{array}$ & $\begin{array}{l}41,92 \\
44,56 \\
61,21 \\
41,11\end{array}$ & $\begin{array}{l}(35,81) \\
(40,67) \\
(49,43) \\
(33,82)\end{array}$ & $\begin{array}{l}30 \\
12 \\
10 \\
23\end{array}$ & $\begin{array}{l}68,89 \\
56,60 \\
76,18 \\
73,18\end{array}$ & $\begin{array}{l}(49,94) \\
(49,35) \\
(61,34) \\
(62,90)\end{array}$ \\
\hline $\begin{array}{l}\text { A nova* } \\
\text { Efeito de } \\
\text { Efeito de } \\
\text { Efeito de }\end{array}$ & & & & \multicolumn{3}{|c|}{$\begin{array}{l}F=0,72 \text { n.s. } \\
F=8,88 \text { p }<0,01 \\
F=0,33 \text { n.s. }\end{array}$} \\
\hline
\end{tabular}

* Análise de variância a dois fatores fixos.

n.s.: não significante $(p>0,05)$. 
RONCADA, M.J. et al. Níveis sangüíneos de vitamina A e caroteno em indivíduos de meiaidade e idosos, em onze localidades do Estado de São Paulo, Brasil. Rev. Saúde públ.. S. Paulo, 19:336-43, 1985.
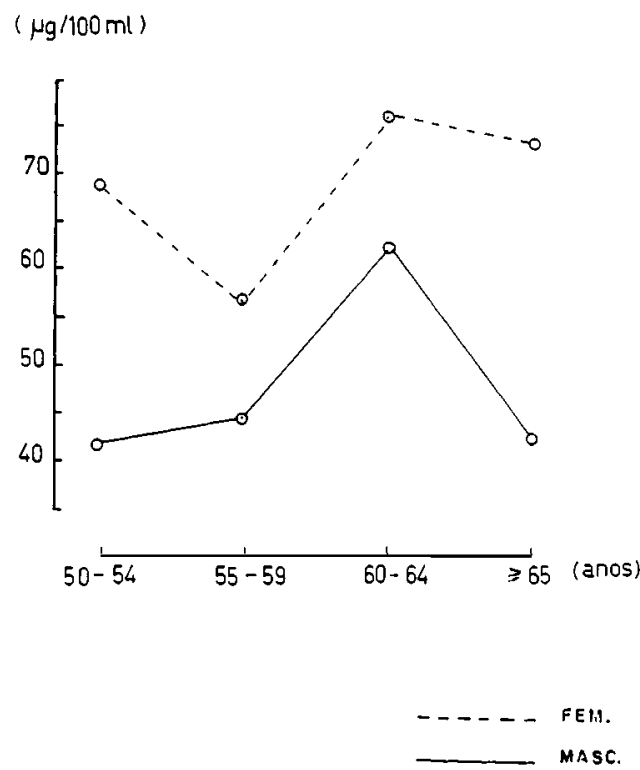

Fig. 2 - Médias das concentraçōes sangüineas de caroteno $(\mu \mathrm{g} / 100 \mathrm{ml})$, segundo sexo e idade, em onze localidades do Estado de São Paulo. 1969-1973.

Entretanto, a análise dos resultados por meio de médias pode trazer informações imprecisas, uma vez que valores aberrantes podem influenciá-las, interferindo na interpretação correta dos resultados. Por isso, optamos por classificar estes últimos segundo os critérios propostos pelo ICNND e caracterizar o problema por meio de proporções de valores dentro ou fora dos limites de normalidade $(20 \mu \mathrm{g} / 100 \mathrm{ml}$ para vitamina $\mathrm{A}$ e $40 \mu \mathrm{g} / 100 \mathrm{ml}$ para caroteno).

Encontramos 3,8\% de resultados de vitamina A plasmática na categoria "deficiente" ( $\leqslant 10 \mu \mathrm{g} / 100 \mathrm{ml}$ ) e $5,8 \%$ na categoria "baixo"; portanto, apenas $9,6 \%$ dos indivíduos estudados estavam abaixo dos limites de normalidade. Assim sendo, essa proporção não caracterizou um problema de Saúde Pública nessa população, com relação à hipovitaminose $\mathrm{A}$.

Comparando com os 3 grupos etários menores de 15 anos (2 a 6,7 a 10 e 11 a 14 anos) estudados simultaneamente nessas 11 localidades ${ }^{19}$, este é um grupo privilegiado, uma vez que, para aqueles, o pcrcentual de resultados abaixo do normal foi, respectivamente, $48,5 \%, 35,0 \%$ e $19,1 \%$.

Embora a proporção de resultados abaixo do normal tenha sido maior entre as mulheres (Fig. 3), a diferença não foi estatisticamente significante, como não o foi também entre os quatro grupos etários em que subdividimos os idosos.

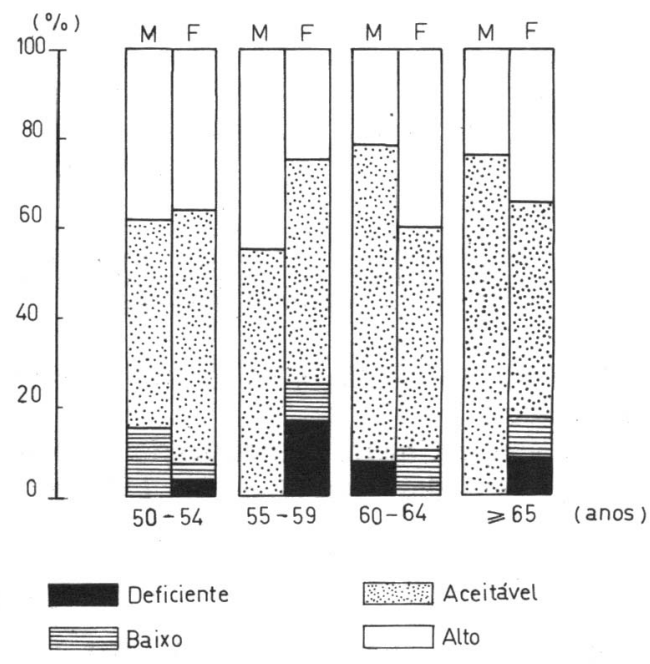

Fig. 3 - Niveis sanguíneos de vitamina A. segundo sexo, idade e classificação do ICNND. em onze localidades do Estado de São Paulo. 1969-1973.

Roncada ${ }^{18}$, ao estudar os níveis das concentrações séricas de vitamina $\mathrm{A}$ e caroteno em 1.097 migrantes nacionais em trânsito pela Capital do Estado de São Paulo, com idades entre 15 e 60 anos, encontrou, em 47 indivíduos de ambos os sexos, de 50 a 60 anos, uma proporção de $21,2 \%$ de resultados abaixo do normal com relação à vitamina $\mathrm{A}$.

Com relação ao caroteno plasmático há que lembrar que os níveis sanguiíneos 
RONCADA, M.J. et al. Níveis sangüíneos de vitamina A e caroteno em indivíduos de meiaidade e idosos, em onze localidades do Estado de São Paulo, Brasil. Rev. Saúde públ., S. Paulo, 19:336-43, 1985.

desse nutriente refletem sua ingestão recente. No presente estudo, encontramos $24,1 \%$ de resultados na classe "deficiente" e $24,7 \%$ na categoria "baixo" $(48,8 \%$ abaixo do normal). Esses números sugerem que a ingestão imediata de alimentos ricos em carotenóides, nesse grupo, foi baixa, talvez devido ao fato de que esses alimentos são, geralmente, frutas ou hortaliças ricas em fibras dietéticas, as quais, provavelmente, foram pouco consumidas por vários motivos, como, por exemplo, deficiência do aparelho mastigador, hábitos sedentários que levam à diminuição do apetite e crenças errôneas sobre ingestão de determinadas frutas em idade avançada; além disso, há que acrescentar que as pessoas idosas muitas vezes vivem sós, o que as leva a hábitos dietéticos incorretos por falta de motivação no preparo de certos alimentos ou mesmo por dificuldades financeiras.

Inversamente do que ocorreu com a vitamina $\mathrm{A}$ sangüínea, os resultados de caroteno abaixo do normal (Fig. 4) foram mais elevados entre os indivíduos do sexo masculino, embora a diferença não tenha sido estatisticamente significante; também não verificamos diferenças entre as proporções encontradas nos quatro grupos etários.

Considerando os resultados obtidos é lícito supor que os niveis elevados de vitamina A plasmática encontrados na população estudada originam-se principalmente do consumo de vitamina A préformada, ou seja, de alimentos de origem animal.

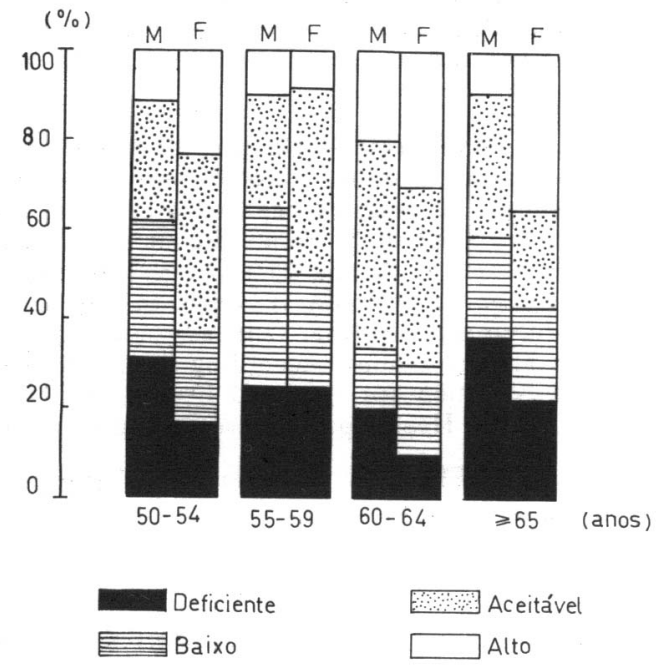

Fig. 4 - Níveis sangǘneos de caroteno, segundo sexo, idade e classificação do ICNND em onze localidades do Estado de São Paulo. 1969-1973.

\section{CONCLUSÕES}

1. A população estudada não apresentou problema de Saúde Pública com relação à hipovitaminose $\mathrm{A}$.

2. Não houve diferenças significantes entre os níveis médios sangüíneos de vitamina $\mathrm{A}$ entre homens e mulheres.

3. As mulheres dos quatro grupos etários tiveram as médias dos niveis sangüíneos de caroteno mais elevadas do que os homens, sendo significativas essas diferenças. 
RONCADA, M.J. et al. Níveis sangüíneos de vitamina A e caroteno em individuos de meiaidade e idosos, em onze localidades do Estado de São Paulo, Brasil. Rev. Saúde públ., S. Paulo, 19:336-43, 1985 .

RONCADA, M.J. et al. [Blood levels of vitamin A and carotene in middle-aged and elderly individuals, in eleven localities of S. Paulo State. Brazil]. Rev. Saúde públ., S. Paulo $19: 336-43$. 1985 .

ABSTRACT: Seeking to fill in a gap in knowledge of vitamin A deficiency regarding biochemical status, carotene and vitamin A blood levels in 158 subjects, male and femals, 50 years of age and over, were analysed. The subjects resided in 11 localities in the State of S. Paulo, Brazil. They were divided into 4 age groups: 50 to 54, 55 to 59,60 to 64 and 65 and over. No significant differences were encountered between averages of vitamin $\mathrm{A}$ levels in the four age groups nor between sexes. However, concerning carotene, females presented significantly higher average levels than males in all age groups. According to the classification of the Interdepartmental Committee in Nutrition for National Defense (ICNND), the population studied did not present a Public Health problem regarding vitamin A status,

UNITERMS: Vitamin A, deficiency. Vitamin A, blood. Carotene, blood. Aged.

\section{REFERENCIAS BIBLIOGRÅFICAS}

1. BAKER, H.; FRANK, O.; THIND, I.S.; JASLOW, S.P. \& LOURIA, D.B. Vitamins profiles in elderly persons living at home or in nursing homes, versus profiles in healthy young subjects. J. Amer. geriat. Soc., 27:444-50. 1970.

2. BASTIAN, E.M. Estudo de aspectos da assistência à saúde da pessoa idosa em instituições não hospitalares do Município de São Paulo. São Paulo, Faculdade de Saúde Pública da USP, 1976. [Mimeografado].

3. BASTIAN, E.M. Estudo sobre acreditação de internatos para pessoas idosas na área metropolitana de Porto Alegre RS. São Paulo, 1979. [Tese de Livre-Docência - Faculdade de Saúde Pública da USP].

4. BOLETIM BIBLIOGRÁFICO. (Ministério da Previdência e Assistência Social/Legião Brasileira de Assistência). Rio de Janeiro, (1) mar. 1982.

5. CAMPBELL, D.A. \& TONKS, E.L. Vita$\min A$, total carotenoids and thymol turbidity levels in plasma. Brit. med. J., 2:1499-501, 1949.

6. CANOAS, C.S. A condição humana do velho. São Paulo, Cortez Ed., 1983.

7. DUCREUZET, C. Le comportement alimentaire des personnes agees. Méd. Nutr., 17:349-52, 1981.
8. FERRARI, M.A.C. Geriatria - aspectos educacionais e de terapia ocupacional. São Paulo, 1975. [Dissertação de Mestrado - Faculdade de Saúde Pública da USP].

9. FERRARI, M.A.C. Idade avançada - nova preocupação de saúde pública. São Paulo, 1981. [Tese de Doutorado - Faculdade de Saúde Pública da USP].

10. GARRY, P.J.; GOODWIN, J.S.; HUNT, W.C.; HOOPER, E.M. \& LEONAR, A.G. Nutritional status in a healthy elderly population: dietary and supplemental intakes. Amer. J. clin. Nutr., 36:319. $31,1982$.

11. GARRY, P.J.; GOODWIN, J.S.; HUNT, W.C. \& GILBERT, B.A. Nutritional status in a healthy elderly population: vitamin C. Amer. J. clin. Nutr., 36:3329, 1982.

12. KERRIGAN, W.M. A Assembléia Mundial sobre o Envelhecimento. Saúde Mundo, p. 7, fev./mar., 1982.

13. KIRK, E. \& CHIEFFI, M. Vitamin studies in middle-aged and old individuals. J. Nutr., 36:315-22, 1948.

14. LAURENTI, R, O envelhecimento da população. Geriat. Sínt., Guarulhos, 1(9): 21-4, 1984. 
RONCADA, M.J. et al. Níveis sangüíneos de vitamina A e caroteno em indivíduos de meiaidade e idosos, em onze localidades do Estado de São Paulo, Brasil. Rev. Saúde públ., S. Paulo, 19:336-43, 1985.

15. MAHLER, H. Remoçando a velhice. In: Organização Mundial da Saúde. Dia Mundial da Saúde de 7 de abril de 1982: remoçar a velhice. Genebra, 1982.

16. MCCLEAN, H.E.; WESTON, R.; BEAVEN, D.W. \& RILEY, C.G. Nutrition of elderly men living alone. I. Intakes of energy and nutrients. $N . Z$. med. J., 84:305-9, 1976.

17. O'HANLON, P. \& KOHRS, M.B. Dietary studies of older Americans. Amer. J. clin. Nutr., 31:1257-69, 1978.

18. RONCADA, M.J. Inquérito entre migrantes atendidos pela Central de Triagem e Encaminhamento, na Capital do Estado de São Paulo, Brasil. II. Aspectos bioquímicos da hipovitaminose A. Rev. Saúde públ., S. Paulo, 9:313-29, 1975.

19. RONCADA, M.J.; WILSON, D.; MAZZILLI, R.N, \& GANDRA, Y.R. Hipovitaminose $\mathrm{A}$ em comunidades do Estado de São Paulo, Brasil. Rev. Saúde públ., S. Paulo, 15:338-49, 1981.

20. ROSS, G. \& PARKER, J.G. Serum carotene zoncentration in normal individuals and its clinical interpretation. $N . Y . J$. Mrd., 62:3584-9, 1962.

21. RUNCIE, J. Nutritional problems in the elderly. Practitioner, 225:1747-52, 1981.

22. SANTOS, L.L.C, de. Açōes de enfermagem e sua importância segundo o enfer- meiro e paciente geriátrico. Florianópolis, 1981. [Dissertação de Mestrado - Univ. Fed. de Santa Catarina].

23. SIEGEL, S. Estatística não-paramétrica. São Paulo, Ed. McGraw-Hill do Brasil, 1981 .

24. THIELE, V.F.; BRIN, M. \& DIBBLE, M.V. Preliminary biochemical findings in negro migrant workers at King Ferry, New York. Amer, J. clin. Nutr., 21: 1.229-38, 1968

25. TODHUNTER, E.N. Nutrition of the elderly. In: Alfin-Slater, R.B. \& Kritchevsky, D. Nutrition and the adultmicronutrients. New York, Plenum Press, 1980, p. 397-417.

26. UNITED STATES. Interdepartmental Committee on Nutrition for National Defense. Manual for nutrition surveys. 2nd ed. Washington, D.C., Government Printing Office, 1963.

27. VIR, S.C. \& LOVE, A.H.G. Nutrition evaluation of $B$ groups of vitamins in institutionalised aged. Int. J. Vit. Nutr. Res., 47:211.8, 1977.

28. WINER, B.J. Statistical principles in experimental design. Tokyo, McGraw-Hill Kogakusha, 1971.

Recebido para publicação em 20/03/1985. Aprovado para publicação em 21/05/1985. 